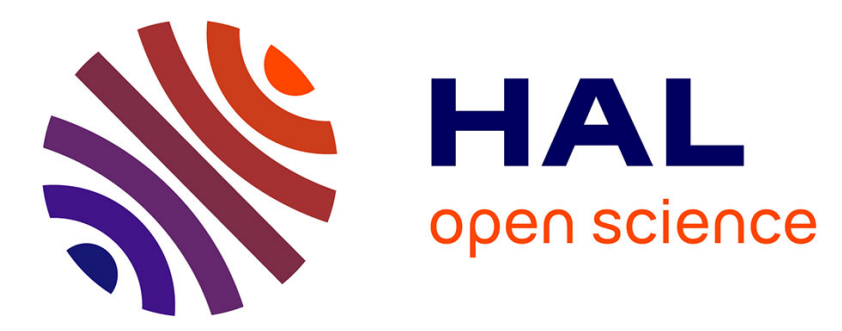

\title{
Autostereoscopic transparent display using a wedge light guide and a holographic optical element: implementation and results
}

Thomas Crespel, Patrick Reuter, Adrian Travis, Yves Gentet, Xavier Granier

\section{- To cite this version:}

Thomas Crespel, Patrick Reuter, Adrian Travis, Yves Gentet, Xavier Granier. Autostereoscopic transparent display using a wedge light guide and a holographic optical element: implementation and results. Applied optics, 2019, 58 (34), 10.1364/AO.58.00G293 . hal-02352672

HAL Id: hal-02352672

https://hal.inria.fr/hal-02352672

Submitted on 6 Nov 2019

HAL is a multi-disciplinary open access archive for the deposit and dissemination of scientific research documents, whether they are published or not. The documents may come from teaching and research institutions in France or abroad, or from public or private research centers.
L'archive ouverte pluridisciplinaire HAL, est destinée au dépôt et à la diffusion de documents scientifiques de niveau recherche, publiés ou non, émanant des établissements d'enseignement et de recherche français ou étrangers, des laboratoires publics ou privés. 


\title{
Autostereoscopic transparent display using a wedge light guide and a holographic optical element: implementation and results
}

\author{
Thomas Crespel $1,2,3,{ }^{*}$, Patrick Reuter $^{1,3,4}$, Adrian Travis ${ }^{6}$, Yves Gentet ${ }^{7}$, And Xavier \\ GRANIER $^{2,5}$ \\ ${ }^{1}$ Inria Bordeaux Sud-Ouest 200 Avenue de la Vieille Tour, 33405 Talence, France \\ 2 LP2N / Institut d'Optique d'Aquitaine, Rue François Mitterrand, 33400 Talence, France \\ ${ }^{3}$ Université de Bordeaux, 351 cours de la Libération, 33405 Talence, France \\ ${ }^{4}$ LaBRI, 351 cours de la Libération, 33405 Talence, France \\ ${ }^{5}$ Archéovision, Archéopôle d'Aquitaine, Esplanade des Antilles, 33607 Pessac, France \\ ${ }^{6}$ Travoptics, 62 avenue de Flandre, 75019 Paris, France \\ ${ }^{7}$ Ultimate Holography, Bordeaux 33800, France \\ *Corresponding author: thomas.crespel@inria.fr
}

Compiled October 3, 2019

\begin{abstract}
We present a novel transparent autostereoscopic display consisting of laser picoprojectors, a wedge light guide, and a holographic optical element. The holographic optical element is optically recorded, and we present the recording setup, our prototype, as well as the results. Such a display can superimpose 3D data on the real world without any wearable. @ 2019 Optical Society of America
\end{abstract}

http://dx.doi.org/10.1364/ao.XX.XXXXXX

\section{INTRODUCTION}

Transparent displays, or see-through displays, allow a user to see the screen while still being able to see what is behind. They offer exciting possibilities for augmented reality as digital content may be superimposed on a real world object or scene. However, the digital content needs to be view-dependent in order to always align on the real objects to augment. Our goal is to provide a transparent display that can be used without tracking or any wearable, with autostereoscopic capabilities. Typical applications would be augmented showcases in museums or shops, head-up displays in vehicles, or any other augmented reality application that would preferably not require the user to wear any headset.

This paper is structured as follows. In Section 2, we review related work on transparent 3D displays and wedge light guides. Section 3 details the general principle of our display. Section 4 focuses on describing the holographic optical element that composes our display. We describe the hardware and software implementation of our prototype in Section 5 and evaluate its performance in Section 6. Section 7 describes the perspective of improvement of our prototype, before we conclude in Section 8 .

This paper is an extended version of a two-page paper presented at the Digital Holography and 3D Imaging conference in May 2019 [1].

\section{RELATED WORK}

\section{A. Transparent and 3D displays}

Transparent displays are possible notably thanks to OLED displays [2] or half-mirror and projector assemblies. However, such displays can generally not be used directly for augmented reality, as the augmentation has to be view-dependent in order to be aligned with the real world content. This problem can be partially solved by tracking the user's eyes positions relatively to the display $[3,4]$. As the user's left and right eye have different positions themselves, a conflict between both eyes may arise. Head-mounted displays (HMDs) do not have the same alignement issues because the eye is fixed relatively to the screen. Indeed, the screen position and orientation must be tracked to update the digital content in real time. Exisiting HMDs can either be monoscopic $[5,6]$ or stereoscopic $[7,8]$.

A conceptually different approach is to simulate a seethrough effect from a video stream and to augment real content on an opaque regular display, like a phone or tablet. These approaches only provide a $2 \mathrm{D}$ projection of both the real and digital world.

Offering stereoscopic images to the user enhances 3D perception by exploiting binocular disparity. The left and right images can be separated towards the left and right eye by letting the user wear active or passive glasses, or the separation can be directly ensured by the display itself. In the latter case, the dis- 
plays are called autostereoscopic. Research on autostereoscopic displays is a broad area, and most of the existing approaches are based on parallax barriers or lenticular arrays $[9,10]$, random holes [11] or compressive light-fields [12]. If more than two views are presented, the display can also showcase other depth cues like motion parallax [13], enhancing even more 3D perception. Autostereoscopic displays often trade spatial resolution for angular resolution [14].

The ability to superimpose stereoscopic images over a realworld scene is an exciting perspective for augmented reality applications. The autostereoscopic displays presented above use conventional optics to separate the views, thus they are hardly adaptable to transparent displays. Takaki et al. [15] adapted the lenticular method to create a see-through flat panel display based on integral imaging with several layers of lenticular arrays and a transparent display, but it suffers from diffraction effects by the pixel pitch and lenticular pitch, as well as low transmittance. The mask-hole method was also adapted to a transparent display [16], but the use of LCD panels inevitably blurs the real objects by diffraction. Hong et al. [17] propose a multi-projection system with a transparent anisotropic diffuser, however, the diffuser is hard to manufacture, and multiprojection in free-space is often unwanted. Lee et al. [18] proposed a compact, nontransparent, multiprojection system using a wedge light guide, a regular vertical diffuser, and Fresnel optics to create multiple viewing zones. Even though the purpose of this research was not to make a see-through display, we can argue that although the light guide is transparent, the Fresnel optics and diffuser distort the real world behind them, so it cannot be directly adapted to a see-through display.

An interesting element to perform view separation while maintaining a good transparency and no distortion is holography, and in particular holographic optical elements (HOEs). For an extensive survey on 3D displays based on holography, we refer the reader to He et al. [19]. Notably, some waveguide-based displays use HOEs, as for example the Hololens [7], where several HOEs are used to perform in-coupling, pupil expansion, and out-coupling in a waveguide [20]. The ability of HOEs to realize almost any optical function makes them suitable for multiprojection systems. Holografika [21] designed a holographic screen for a full parallax opaque display using a dense projector arrangement. Olwal et al. [22] designed the ASTOR display with two projectors in free-space and a reflective holographic combiner that scatters the light of each projector in separate viewing zones. Their display also involves free-space projection, and this may be unwanted for several reasons: the whole resulting system is bulky and not standalone as projectors must be positioned in accordance with the screen location, and the projection may be subject to occlusion issues. The fabrication of the involved HOEs is often eluded in the aforementioned literature, and our intention is to clearly describe also the recording process.

\section{B. Wedge light guides}

Our display involves a a wedge light guide to hold the projection distance, and we describe related work for such waveguides in the following.

Wedge optics have been widely used, and the most used case is wedge prisms to deflect or shape beams. Wedge light guides are specific wedge optics that were first developed for flat panel projection devices [23] and later adapted to a flat panel timemultiplexed 3D display with a LED backlight and a LCD [24].

As depicted in Figure 1, a wedge light guide is roughly composed of two parts: an expansion part and a wedge part. If a

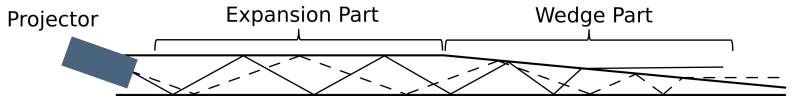

Fig. 1. Principle of a wedge guide

ray is shone into the entrance of the expansion part, it will be guided through total internal reflection towards the wedge part, where one interface is tilted relatively to the other. The rays will then undergo further total internal reflection bounces but the angle of reflection lowers at each bounce until the critical angle is reached, and the ray escapes the guide.

If a projector launches a fan of rays, then each ray will escape at a different position depending on the input angle: in the plane of the drawing, the grazier the angle, the further it will propagate. The image carried by the projector diverges in the plane of the guide in the expansion part, and the wedge part realizes the expansion of the image along the direction of propagation.

If a diffuser is located at the extraction surface, the projected image will be visible to a user like in free-space projection and without the need to couple out the beam from the guide.

If nothing blocks or redirects the rays at the exit surface, then they will simply continue their propagation. When the rays have just escaped the total internal reflection regime, they will leave the guide with an angle close to $90^{\circ}$ with respect to the normal of the exit surface in the plane of the drawing, while they keep on diverging from a point source in a direction parallel to the plane. The shape of a beam created by a projector and escaping a wedge guide is then collimated in one direction and diverging in the other, and propagates with a grazing angle with respect to the wedge surface [25]. Readers must be aware that unlike the wedge guide described in [18] and schematically drawn in Figure 1, these wedge guides do not have rigorous planar faces but have a thickness profile that is optimized to force rays to undergo the same number of reflections and leave the guide from the same side [26]. The advantage of a wedge guide compared to a regular slab is that there is no need for incoupling, pupil expansion nor out-coupling, as the guide itself ensures these functions.

Our main contribution in this paper compared to other work on wedge guides is to replace the regular diffuser that scatters the light from one projector uniformly by a holographic diffuser that scatters the light from several projectors to separate viewing zones.

\section{OVERVIEW}

The principle of our display, as depicted in Figure 2, is to couple beams from multiple laser beam steering picoprojectors into a transparent wedge guide and then to redirect each beam to separate viewing zones using a transparent $\mathrm{HOE}$.

The viewing zones have a width $W_{V Z}$ and a height $H_{V Z}$, and they are located at a distance $D_{o b s}$ in front of the HOE. If $W_{V Z}$ is an integer fraction of the interpupillary distance $d_{I P D}$, then a user located at $D_{o b s}$ in front of the display has both eyes in separate viewing zones and perceives a stereoscopic image with naked eyes.

\section{HOLOGRAPHIC OPTICAL ELEMENT}

\section{A. Role of the HOE}

A transmission volume $\mathrm{HOE}$ is clamped to the wedge exit surface in order to create the viewing zones. Note that the HOE is 


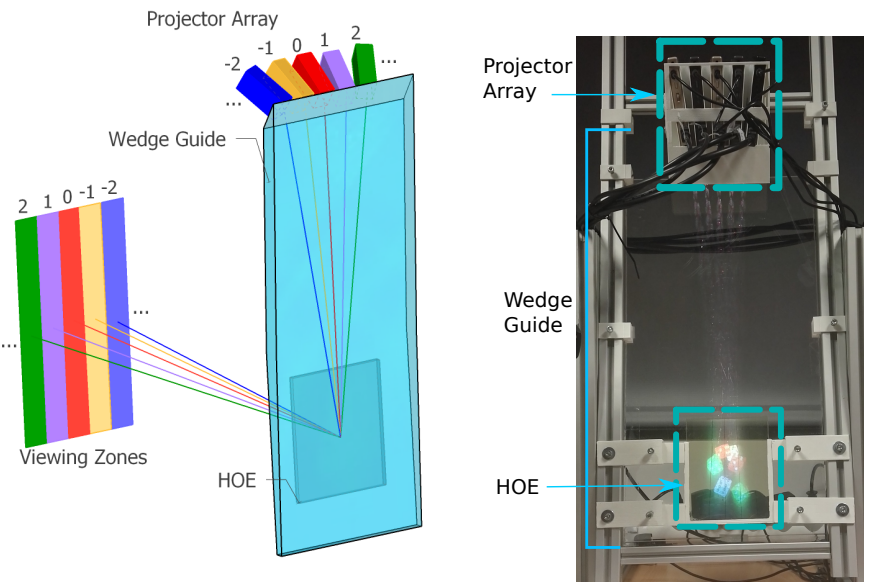

Fig. 2. (left) Overview of the prototype: 5 projectors labeled $-2 . .2$ are coupled into a wedge light guide, and a HOE scatters the light towards independent viewing zones -2...2. (right) Photograph of our prototype from viewing zone 0

not optically coupled with the wedge guide: a tiny air interface ensures that rays propagate and leave the guide as described in Section 2B. The role of the HOE is to scatter the light from each projector to an independent viewing zone: referring to Figure 2 , the light projected by projector $i$ must be only visible in the viewing zone $i$ (with $i=-2.2$ ).

Our approach is the following: we record the optical function for the central viewing zone only as we explain in Section B. In addition, as we detail in Section C, we use the large angular bandwidth of the hologram to reconstruct the other viewing zones.

\section{B. Recording setup}

The HOE is optically recorded, on the material Ultimate 04 (U04) from Ultimate Holography [27], and so we need to create an interference pattern between a reference beam and an object beam. Let us first consider the recording for the central projector position $(i=0)$. Figure 3 illustrates the recording setup and the coordinate systems used for the following explanations.

The object beam is generated by a rectangular diffuser of size $W_{V Z} \times H_{V Z}$ and located at $D_{o b s}$ in front of the hologram, and this corresponds to the desired properties of the viewing zone. To uniformly light the diffuser, we first spread the beam with a cylinder lens L2. Its focal length does not matter since the objective is simply to create a line on a first diffuser. This line is scattered toward a second diffuser that represent the recorded object, and each point of it is then scattered toward the full hologram.

Defined this way, it is clear that the reconstruction has to result in a real image of the object where rays converge, rather than a virtual image. Thus, the reference beam must be the complex conjugated of the incident beam, i.e. the counterpropagating wave.

The beam that is incident on the HOE is the beam that exits the wedge guide: it is collimated in the plane of Figure 1 and diverging in a plane parallel to the wedge (see Section 2 and [25]), so the reference beam must be collimated and converging. Moreover, it must be incident on the hologram with a grazing angle corresponding to the exit angle of the wedge guide $\left(\beta \approx 8^{\circ}\right)$.

To create the reference beam, we first spread it by a spatial

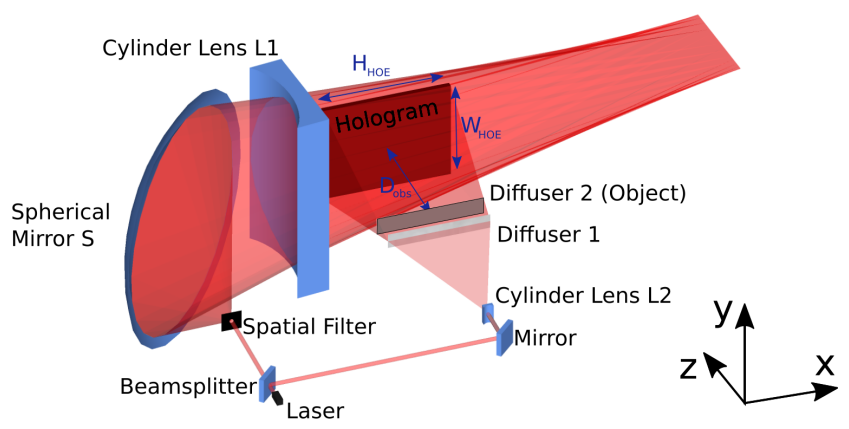

(a) Side view

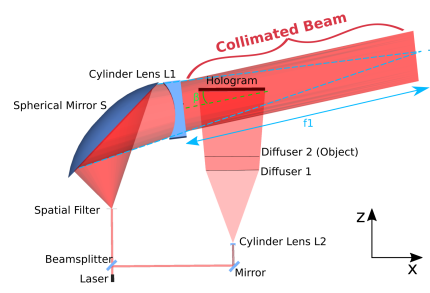

(b) Top view

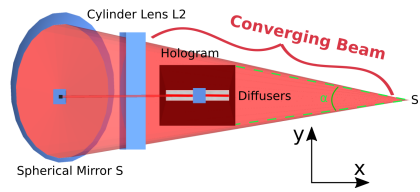

(c) Front view
Fig. 3. Recording setup of the HOE. The object beam is created by a rectangular diffuser representing the viewing zone. The reference beam is created by creating a convergent beam with a spherical mirror and collimate it in one direction with a cylinder lens.

filter toward a spherical mirror. As the mirror is in off-axis configuration, the beam is converged into a sagittal point $S$ and tangential point $\mathrm{T}$ due to astigmatism. The center of the hologram is located at about $400 \mathrm{~m}$ from $\mathrm{S}$ so that the converging angle $\alpha$ corresponds to the diverging angle from the wedge at the HOE location $\left(\alpha \approx 15^{\circ}\right)$. On the other hand, we add a diverging cylinder lens L1 of focal length $-500 \mathrm{~mm}$ so that the tangential image is located at its focal plane and the beam gets collimated in this direction. The lens L1 must be sufficiently high so that the converging beam covers entirely the HOE and in practice, we glued 8 lenses together to obtain a $200 \mathrm{~mm}$ high lens.

We have recorded a monochromatic green $\mathrm{HOE}$ and a color HOE. The color HOE is obtained with wavelength multiplexing, and the laser beam shown in Figure 3 is composed of red, green, and blue lasers.

\section{Reconstruction of the adjacent viewing zones}

Our HOE is a volume hologram, therefore it has Bragg selectivity, and in particular angular selectivity. The typical efficiency curve [28] with respect to the deviation from the Bragg angle is shown in Figure 4. Note that the Bragg angle is different for each position of the hologram but every point exhibits this angular efficiency.

Rays that are incident on the HOE with the Bragg angle (the recording angle) create a viewing zone with maximum efficiency.

Rays whose angles belong to the angular bandwidth still reconstruct a viewing zone but with a lower efficiency and we use this range to reconstruct the other viewing zones. The dashed lines illustrate where the angle of incidence of each projector are located with respect to the Bragg angle and bandwidth. The position of the resulting viewing zone is shifted with respect to the input angular shift, so it is possible to arrange the projectors 


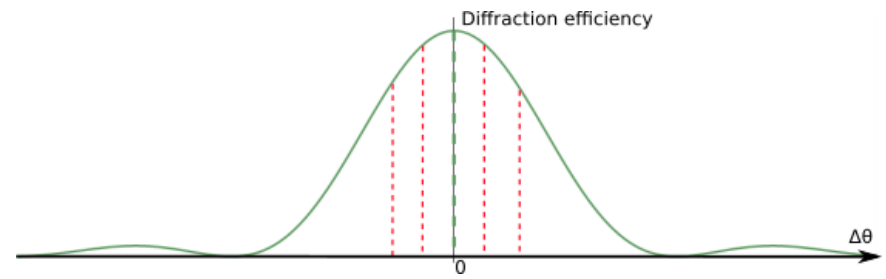

Fig. 4. Typical diffraction efficiency of a volume hologram depending on the deviation from the Bragg angle and the incidence angles of the 5 projectors of our display (in dashed lines)

so that the viewing zones are adjacent to each other.

Rays that are incident on the hologram with an angle outside the angular bandwidth are not diffracted. This is the case with light that does not come from the inside of the wedge, thus the HOE appears transparent in a natural environment.

\section{Alternative approach}

It could have been possible to have a narrower angular bandwidth and to angular-multiplex as much gratings as projectors, but the recording setup would have been much more complicated, and the diffraction efficiency potentially lower. Here, we show that it is possible to record a hologram for a single projector and use the large bandwidth to reconstruct the other viewing zones, without angularly multiplexing each projector position. This also has the advantage of giving more freedom on the positioning of the projectors after the recording process.

\section{PROTOTYPE OF THE DISPLAY}

\section{A. Hardware design}

We use an off-the-shelf wedge guide designed according to patent [26], with a size of $480 \mathrm{~mm} \times 250 \mathrm{~mm}$ and a thickness varying from $10 \mathrm{~mm}$ to $1 \mathrm{~mm}$. The HOE is located at the imaging area of the wedge guide. We use $N_{\text {proj }}=5$ Sony MP-CL1A laser picoprojectors [29] that are connected to a laptop computer with an external graphical card. Each projector is separated by its neighbor(s) by $17 \mathrm{~mm}$, and they converge toward the HOE. The viewing zones are $W_{V Z}=30 \mathrm{~mm}$ wide and $H_{V Z} \approx 100 \mathrm{~mm}$ high and are located at $D_{o b s}=50 \mathrm{~cm}$. The prototype is built thanks to an aluminium frame and 3D printed elements.

\section{B. Software Calibration}

Our software generates $N_{\text {proj }}$ renderings of a 3D scene and casts them to $N_{\text {proj }}$ fullscreen windows on the projectors, which are connected as external monitors. The images must be aligned with each other on the plane of the HOE, and this is done by correcting both the wedge optical distortion and the positional relative projectors' offset.

To this end, we go through a calibration procedure that has to be done only once (provided that the prototype elements remain fixed between each other). The calibration procedure is illustrated in Figure 5 and described below:

1. We replace the HOE with a regular diffuser (e.g. a piece of paper), so that the images projected by each projector are all visible from a single viewpoint.

2. A distortion-corrected camera images the diffuser, and the area to be calibrated has to be cropped.

3. A regular grid is drawn in camera space.

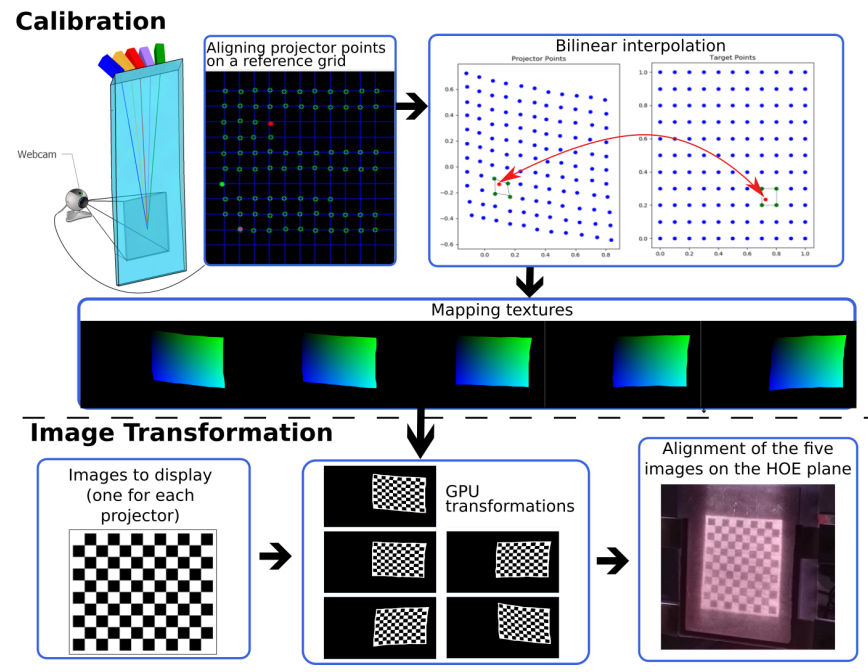

Fig. 5. Calibration procedure and image transformation of 5 checkerboard patterns that align on the imaging area

4. An algorithm projects a point in projector space and moves it until it matches a target corner in the camera grid. This is illustrated in Figure 5 where three points are projected and tracked by their color simultaneously.

5. We then extrapolate the calibrated points to a texture of the size of the projector resolution: for each pixel, we perform a bilinear interpolation in a quad formed by the closest calibrated points.

We call the resulting texture of this preprocess the mapping texture, and it stores for each projector pixel the corresponding coordinate of the target texture. When using our display, all the projected images are corrected in real time on the GPU: for each projector pixel, we look up the value of the mapping texture which provides the pixel coordinates to read in the input image.

We evaluate the required image transformation without any a priori knowledge on projector position or angle, or on the wedge guide properties. Our algorithm takes two minutes on average to calibrate one projector. Figure 5 shows the deformation of 5 checkerboard images that align on the imaging area.

\section{RESULTS}

In this section, we evaluate the performance of our display. We first focus on the practical reconstruction of the viewing zones with the monochromatic HOE in Section A. We then describe the color issues that we observed with the full-color HOE in Section B, before evaluating the display as a whole in Section C.

\section{A. Reconstruction of the viewing zones}

The five projectors are located at the entrance of the wedge guide and display fullscreen green-only images. The monochromatic HOE is located at the wedge part of the guide and the reconstruction of the viewing zones on a $D_{o b s}$ distant screen can be seen in Figure 6(a). One can observe that they are well defined, and their size match the size of the recorded object. Note that the full-color HOE also shows the same behavior, except for color issues that are specifically addressed in Section B.

We insist on the fact that this rectangular diffuser is reconstructed with a projector that fully lights the HOE, and if only a portion of it is lit (e.g. one pixel), then the entire viewing zone will be reconstructed as well, with a much lower intensity. 


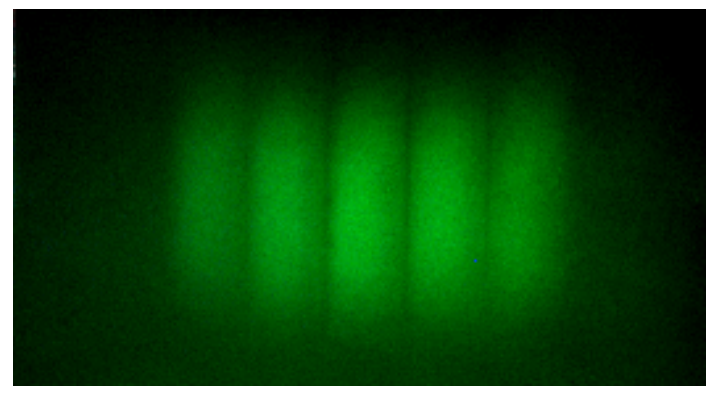

(a)

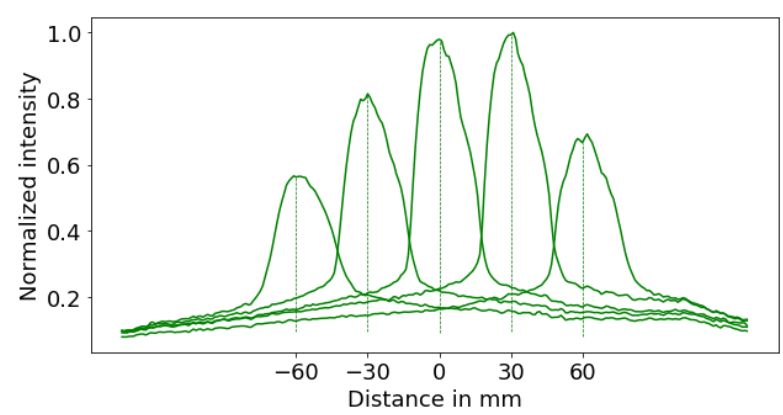

(b)

Fig. 6. (a) Photograph of the 5 views projected on a screen at distance $d_{o b s}$ from the HOE; (b) Normalized intensity profile along the horizontal direction

Figure 6(b) shows a transverse profile of Figure 6(a), showing the relative intensity of each viewing zone. As predicted by the coupled-wave theory [28], the intensity lowers from the central positions with a $\sin c^{2}$ modulation when the angle of incidence diverges from Bragg angle.

The outermost projectors have an efficiency ratio of $60 \%$ with respect to the central one. Hence, the number of projectors $N_{\text {proj }}$ can be more than 5 before extinction of the diffraction efficiency. We evaluate at $N_{\text {proj,max }}=9$ the maximum number of projectors with a minimum intensity ratio of $20 \%$ with respect to the central one. Uniformity in intensity can be achieved computationally by lowering the intensity of the brightest viewing zones so that they match the darkest one.

\section{B. Color issues}

The full-color HOE also reconstructs the viewing zones with the same behavior and angle dependency as explained in the previous section. In addition, each viewing zone display chromatic issues that are specifically discussed in this section.

Figure 7(a) shows a picture of a viewing zone created on a $D_{o b s}$ distant screen by a fullscreen white image on the projector. Figures 7(b), 7(c), and 7(d), respectively, show the contributions of the red, green and blue wavelengths.

Figure 7(e) illustrates the wavelength-dependent contributions to the final result:

- The images $R_{R}, G_{G}$ and $B_{B}$ are sharply imaged and superimposed at the screen position, as desired. An eye located in this area can thus perceive a full color image. The ideal white balance can be achieved either by software or by optimizing exposure times for each wavelength at the recording step.

- The red wavelength does reconstruct any other image.

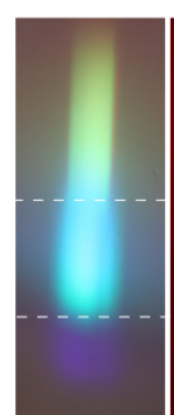

(a)

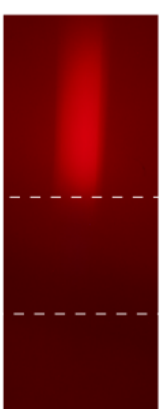

(b)

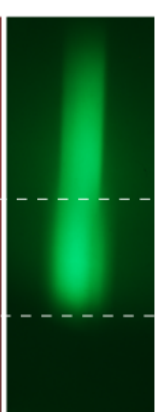

(c)

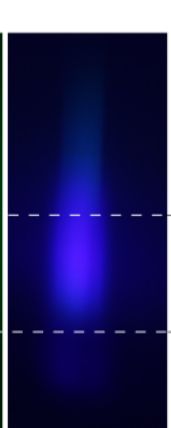

(d)

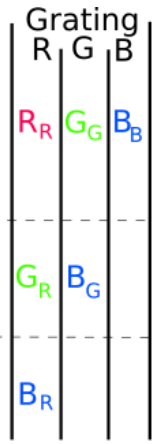

(e)
Fig. 7. Images of the viewing zones on a $D_{o b s}$ distant screen, created by (a) Red, green and blue wavelengths; (b) Red only; (c) Green only; (d) Blue only; (e) Illustration of the wavelengthdependent contributions of each grating.

- The areas $G_{R}$ and $B_{G}$ are overlapping and appear blurred at the screen location. They are actually images that are reconstructed $15 \mathrm{~cm}$ further than $D_{o b s}(65 \mathrm{~cm}$ away from the HOE). These images are undesired crosstalk images that are respectively created by the green wavelength diffraction in the red grating, and the blue wavelength diffraction in the green grating.

- The image $B_{R}$ is located $135 \mathrm{~cm}$ in front of the HOE and corresponds to the order created by the blue wavelength diffraction in the red grating.

At the time of the recording, we did not measure the wavelength dependency of the diffraction efficiency. Finally, the red grating appears to be more efficient than the green one, which is itself more efficient than the blue. This explains why the areas $G_{R}$ and $B_{G}$ are brighter than $G_{G}$ and $B_{B}$, respectively.

We measure the RGB area to be about $75 \mathrm{~mm}$ high, and this is the effective viewing zone size where an eye can perceive a color image. In the current state of the prototype, if an eye is located too low in the eyebox, the user would see an image that does not contain red. We discuss in Section 7 how to avoid such color effects.

\section{Display}

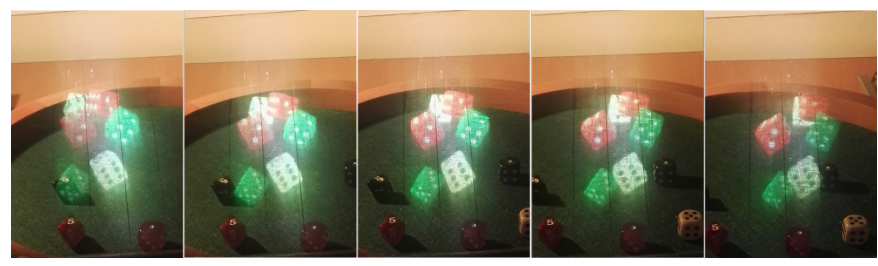

Fig. 8. Photographs of the display from each viewing zone. Parallax and transparency can be obsesrved.

Figure 8 shows five photographs of the display taken from each viewing zone, showing full color and transparency. The images are rendered with a slightly different perspective and corrected with the procedure described in Section B. As a consequence, binocular disparity and horizontal motion parallax are rendered and allow to have a good appreciation of the depth of the scene. We observed that a tolerance of about $\pm 10 \mathrm{~cm}$ around $D_{o b s}$ is acceptable to view the entire images, and the measured 
field of view is $17^{\circ}$. Small vertical black lines are visible, and they are due to the cylinder lens that we made by gluing smaller lenses together: the interfaces have cast shadows on the hologram. They can be easily removed by using a single lens instead of glued lenses during the recording.

\section{FUTURE WORK}

\section{A. Improvements on the HOE}

The most required improvement of the HOE is the correction of chromatic aberrations described in Section 6, and several approaches are possible. We could use the same material and design a solution based on the observations. For example, recording another red viewing zone that overlaps with $G_{R}$ and $B_{G}$ would allow to see color images in this area. Simply extending the size of the viewing zone in this direction can also reduce the probability of being in the wrong location. Another way of improving color rendering would be to increase the thickness of the hologram to reduce spectral bandwidth and avoid color crosstalk. The problem with this approach is that the angular bandwidth would be reduced as well, and the maximum angle of incidence separating the outermost projectors would be lower. With constant projector pitch, this implies that $N_{\text {proj }}$ would be lower, and the field of view would also be smaller. With this approach, it could be interesting to have a significantly narrower bandwidth and multiplex the hologram for each projector position.

If color aberrations remain, a software white balance based on eye tracking should decrease perceived color shifting as long as red, green, and blue are in all viewing zones.

Depending on the application, it could be interesting to study how the image plane could be relocated behind the HOE plane, so that it is closer to the real objects and thus reduces the accomodation-vergence conflict for augmented reality applications. A possible solution might be to put an imaging lens between the diffuser and the hologram in the recording setup. In further research, we might study as well the recording of multiple imaging planes.

For now, the HOE has a size of $10 \times 13 \mathrm{~cm}$, but for some applications it might be interesting to record a bigger HOE. The process could be a bit more complicated because of the larger optics that would be required to create the converging beam.

The other parameters of the HOE recording setup can also be changed according to the targeted application: $D_{o b s}$ can be changed at will, and $W_{V Z}$ can be decreased to produce a denser horizontal parallax with denser image sources as well.

\section{B. Improvements on the display}

Apart from the HOE, several perspectives of improvement on the other parts of the display will be investigated.

First of all, eye tracking can improve the 3D depth cues by updating the displayed images with respect to the position of the user's eyes. Without tracking, the digital scene perspective does not change when moving horizontally in the $W_{V Z}$ range, and vertically in the $H_{V Z}$ range. Eye tracking could improve the motion parallax feeling and reduce the transition effects between views while still producing stereoscopic images.

Adding more projectors up to $N_{\text {proj,max }}$ would improve the field of view from $17^{\circ}$ to $30^{\circ}$ and offer more viewing zones. However, having a lot of projectors might not be a practical solution due to the cost of the system and the consumed power. It should be possible to have an opto-mechanical system to rasterize several virtual projector positions at high frequency and synchronize the rendering with it.

Finally, note that we used an off-the-shelf wedge guide that was not originally designed for this application, and it might be interesting to manufacture a dedicated wedge guide that is optimized for several projector positions. In particular, having a curved entrance instead of a rectangular one should improve the reconstruction quality by ensuring that the distance between the light source and the hologram is constant. Changing the size of the wedge guide with respect to the targeted application and HOE size should also be investigated. In particular, there is no physical limitation to design a phone-sized display.

\section{CONCLUSION}

We have demonstrated the feasibility of a compact, autostereoscopic transparent display with multiple projectors and a custom HOE. The HOE is wavelength-multiplexed for full-color efficiency, but only one angular grating is recorded and multiple viewing zones are reconstructed with several projector positions due to the high angular bandwidth. Although some chromatic issues are visible, they can be corrected and the proof-of-concept is demonstrated.

Our current prototype has $N_{\text {proj }}=5$ views but is theoretically able to generate up to $N_{\text {proj,max }}=9$ views.

The views are located $50 \mathrm{~cm}$ in front of the display, they are $3 \mathrm{~cm}$ wide and $10 \mathrm{~cm}$ high. These values are fixed once the HOE is recorded; they result from our choices and can be changed in the recording step. Having narrower viewing zones would give a denser horizontal parallax but would also require to have as much more projectors. Eye tracking can improve horizontal motion parallax and add vertical motion parallax.

This display has great potential for augmented reality applications such as augmented exhibitions in museums or shops, head-up displays for vehicles or aeronautics, and industrial maintenance, among others.

\section{REFERENCES}

1. T. Crespel, P. Reuter, X. Granier, and A. Travis, "Autostereoscopic transparent display using a wedge light guide and a holographic optical element," in Digital Holography and Three-Dimensional Imaging 2019, (Optical Society of America, 2019), p. M3A.4.

2. P. Gorrn, M. Sander, J. Meyer, M. Kroger, E. Becker, H.-H. Johannes, W. Kowalsky, and T. Riedl, "Towards see-through displays: Fully transparent thin-film transistors driving transparent organic light-emitting diodes," Adv. Mater. 18, 738-741 (2006)

3. J. Lee, A. Olwal, H. Ishii, and C. Boulanger, "Spacetop: Integrating 2d and spatial 3d interactions in a see-through desktop environment," in Proceedings of the SIGCHI Conference on Human Factors in Computing Systems, (ACM, New York, NY, USA, 2013), CHI '13, pp. 189-192.

4. V. S. Popescu and J. P. Wachs, "Simulated transparent display with augmented reality for remote collaboration," (2016).

5. Optinvent (2019 (accessed 8th July 2019)). www.optinvent.com.

6. Google Glass. www.google.com/glass/start.

7. Microsoft Hololens (2019 (accessed 8th July 2019)). www.microsoft. com/hololens.

8. Meta (2016 (accessed 8th July 2019)). www.metavision.com.

9. D. E. Roberts, "History of lenticular and related autostereoscopic methods," Leap Technol. Hillsboro 16 (2003).

10. Alioscopy. www.alioscopy.com (accessed 11th July 2019).

11. A. Nashel and H. Fuchs, "Random hole display: A non-uniform barrier autostereoscopic display," in 2009 3DTV Conference: The True VisionCapture, Transmission and Display of 3D Video, (IEEE, 2009), pp. $1-4$. 
12. G. Wetzstein, D. Lanman, M. Hirsch, and R. Raskar, "Tensor Displays: Compressive Light Field Synthesis using Multilayer Displays with Directional Backlighting," ACM Trans. Graph. (Proc. SIGGRAPH) 31, 1-11 (2012).

13. I. P. Howard and B. J. Rogers, Seeing in depth, Vol. 2: Depth perception. (University of Toronto Press, 2002).

14. T. Jarvenpaa and M. Salmimaa, "Optical characterization of autostereoscopic 3-d displays," J. Soc. for Inf. Disp. 16, 825-833 (2008).

15. Y. Takaki and Y. Yamaguchi, "Flat-panel see-through three-dimensional display based on integral imaging," Opt. Lett. 40, 1873-1876 (2015).

16. A. Karnik, W. Mayol-Cuevas, and S. Subramanian, "Mustard: A multi user see through AR display," in Proceedings of the SIGCHI Conference on Human Factors in Computing Systems, (ACM, New York, NY, USA, 2012), CHI '12, pp. 2541-2550.

17. J.-Y. Hong, S.-G. Park, C.-K. Lee, S. Moon, S.-J. Kim, J. Hong, Y. Kim, and B. Lee, "See-through multi-projection three-dimensional display using transparent anisotropic diffuser," Opt. Express 24, 14138-14151 (2016).

18. C.-K. Lee, S. gi Park, S. Moon, J.-Y. Hong, and B. Lee, "Compact multiprojection $3 d$ display system with light-guide projection," Opt. Express 23, 28945-28959 (2015).

19. Z. He, X. Sui, G. Jin, and L. Cao, "Progress in virtual reality and augmented reality based on holographic display," Appl. Opt. 58, A74A81 (2019).

20. B. C. Kress and W. J. Cummings, "11-1: Invited paper: Towards the ultimate mixed reality experience: Hololens display architecture choices," SID Symp. Dig. Tech. Pap. 48, 127-131 (2017).

21. T. Balogh, T. Forgács, T. Agács, O. Balet, E. Bouvier, F. Bettio, E. Gobbetti, and G. Zanetti, "A scalable hardware and software system for the holographic display of interactive graphics applications." in Eurographics (Short Presentations), (2005), pp. 109-112.

22. A. Olwal, C. Lindfors, J. Gustafsson, T. Kjellberg, and L. Mattsson, "Astor: An autostereoscopic optical see-through augmented reality system," in Proceedings of the 4th IEEE/ACM International Symposium on Mixed and Augmented Reality, (IEEE Computer Society, 2005), pp. 24-27.

23. A. Travis, F. Payne, F. Zhong, and J. Moore, "Flat panel display using projection within a wedge-shaped waveguide," in Proceedings of the 20th International Display Research Conference, vol. 2000 (Society for Information Display (SID), 2000), pp. 292-295.

24. C. Lee, A. Travis, and R. Lin, "Flat-panel autostereoscopic 3d display," Optoelectronics, IET 2, $24-28$ (2008).

25. T. Crespel, A. Travis, P. Reuter, and X. Granier, "Wedge cameras for minimally invasive archaeology," J. Comput. Cult. Herit. 12, 14:1-14:13 (2019).

26. A. Travis, "Flat-panel display using tapered waveguide," (2003). Patent WO/2003/013151.

27. Ultimate Holography (2019 (accessed 8th July 2019)). www. ultimate-holography.com.

28. H. Kogelnik, Coupled Wave Theory for Thick Hologram Gratings (Nokia Bell Labs, 1969), pp. 133-171.

29. Sony MP-CL1A (2017 (accessed 8th July 2019)). www.sony.com/ electronics/projector/mp-cl1a. 